\title{
Extracellular Matrix-dependent Pathways in Colorectal Cancer Cell Lines Reveal Potential Targets for Anticancer Therapies
}

\author{
VAIDOTAS STANKEVICIUS ${ }^{1,2}$, GINTAUTAS VASAUSKAS ${ }^{1,2}$, RIMANTE NOREIKIENE ${ }^{1,2}$, \\ KAROLINA KUODYTE ${ }^{1,2}$, MINDAUGAS VALIUS ${ }^{3}$ and KESTUTIS SUZIEDELIS ${ }^{1,2}$ \\ ${ }^{1}$ Laboratory of Molecular Oncology, National Cancer Institute, Vilnius, Lithuania; \\ ${ }^{2}$ Department of Biochemistry and Molecular Biology, Faculty of Natural Sciences, Life Sciences Center, and \\ ${ }^{3}$ Proteomics Center, Vilnius University Institute of Biochemistry, \\ Life Sciences Center, Vilnius University, Vilnius, Lithuania
}

\begin{abstract}
Background: Cancer cells grown in a $3 D$ culture are more resistant to anticancer therapy treatment compared to those in a monolayer $2 D$ culture. Emerging evidence has suggested that the key reasons for increased cell survival could be gene expression changes in cell-extracellular matrix (ECM) interaction-dependent manner. Materials and Methods: Global gene-expression changes were obtained in human colorectal carcinoma HT29 and DLD1 cell lines between $2 D$ and laminin-rich (lr) ECM $3 D$ growth conditions by gene-expression microarray analysis. The most significantly altered functional categories were revealed by Kyoto Encyclopedia of Genes and Genomes (KEGG) pathway enrichment analysis. Results: The microarray data revealed that 841 and 1190 genes were differentially expressed in colorectal carcinoma DLDI and HT29 cells. KEGG analysis indicated that the most significantly altered categories were cell adhesion, mitogen-activated protein kinase and immune response. Conclusion: Our results indicate altered pathways related to cancer development and progression and suggest potential ECM-regulated targets for the development of anticancer therapies.
\end{abstract}

Traditionally, the most common cancer cell-based in vitro assays are performed using 2D cell cultures, often growing on plastic substrates. Although data obtained using 2D cell cultures have revealed critical knowledge related to the complex nature of tumor cells, monolayer cell cultures

Correspondence to: Kestutis Suziedelis, Laboratory of Molecular Oncology, National Cancer Institute, Santariskiu str. 1, Vilnius 08660, Lithuania. Tel: +370 52190904, Fax: +370 52720164, e-mail: kestutis.suziedelis@nvi.lt

Key Words: 3D Cell culture models, transcriptomics, KEGG analysis. poorly mimic biological processes occurring in tumor tissue due to the artificial environment and standardized growing conditions (1). Moreover, important cell functions such as proliferation or morphology can be dramatically altered in 2D cell cultures (2).

The extracellular matrix (ECM), as a key component of the tumor microenvironment, has a high impact on tumor development and cancer cell features (3). The ECM not only structurally supports cancer cells, but also affects other cellular functions, such as cell differentiation, migration, survival or proliferation (4-7). Moreover, gene and protein expression levels are regulated in a cell-ECM interactiondependent manner (8). The ECM is also implicated in tumor cell response to external stimuli (9). Hence, an ECM-based (3D) cell culture, as a model closer to tumor tissue, has been suggested to represent tumor properties better than $2 \mathrm{D}$ cell culture does.

Previous studies have shown that the morphology of cancer cells cultivated in 2D and ECM-based 3D cultures can differ dramatically (10). Cells grown in a 3D culture are also more resistant to anticancer therapy treatment compared to those in a monolayer culture $(11,12)$. Emerging evidence suggests that the key reasons for increased cell survival or reduced cell death could be changes in gene expression in cell-ECM interaction-dependent manner (13). Analysis of ECM dependent gene-expression changes is expected to reveal potential targets for more efficient anticancer therapies. Therefore, in order to investigate which cellular pathways that are altered in an ECM-dependent manner are potential targets for the development of new anticancer therapy strategies and in order to establish a model for these investigations, we compared genome-wide transcriptome changes of two human colon carcinoma cell DLD1 and HT29 lines grown in laminin-rich (lr) ECM 3D cell culture compared to conventional 2D monolayer conditions following $48 \mathrm{~h}$ of cultivation. 
Table I. Primer sequences used in quantitative real time polymerase chain reaction.

\begin{tabular}{lll}
\hline Gene & Forward primer & Reverse primer \\
\hline$M Y B$ & GAAAGCGTCACTTGGGGAAAA & TGTTCGATTCGGGAGATAATTGG \\
$I D 1$ & GGCTGTTACTCACGCCTCAAG & CCAACTGAAGGTCCCTGATGTAG \\
$I D 2$ & TGAGGTCCGTTAGGAAAAACAG & GATTTCCATCTTGCTCACCTTC \\
$I D 3$ & TCACTGTAGCGGGACTTCTTTT & CTGTAGGATTTCCACCTGGCTA \\
$C D K N 1 C$ & CAGAACCGCTGGGATTACGA & CACCGAGTCGCTGTCCACTT \\
$H P R T 1$ & TGCAGACTTTGCTTTCCTTGGTC & CCAACACTTCGTGGGGTCCTT \\
\hline
\end{tabular}

\section{Materials and Methods}

Cell culture and maintenance. Human colon carcinoma cell line DLD1 and HT29 cells were obtained from the American Type Culture Collection (Rockville, MD, USA). For the 2D cell culture, cells were cultured in Dulbecco's modified Eagle's medium (DMEM) (HT29 cell line) or RPMI-1640 (DLD1 cell line) cell culture medium (ThermoFisher Scientific, Waltham, MA, USA) supplemented with $10 \%$ fetal bovine serum (ThermoFisher Scientific), $2 \mathrm{nM}$ glutamine (ThermoFisher Scientific), $100 \mathrm{UI} / \mathrm{ml}$ penicillin (ROTH, Karlsruhe, Germany) and $0.1 \mathrm{mg} / \mathrm{ml}$ streptomycin (ROTH) at $37^{\circ} \mathrm{C}$ in a humidified atmosphere containing $5 \% \mathrm{CO}_{2}$. For the $3 \mathrm{D}$ cell culture, cells were plated in $0.5 \mathrm{mg} / \mathrm{ml}$ Ir-ECM $\left(\right.$ Geltrex $^{\mathrm{TM}}$, ThermoFisher Scientific) and cell culture medium upon a layer of agarose in 24-well cell-culture plates as described elsewhere (14). For all experiments, cells were harvested after $48 \mathrm{~h}$ of cultivation.

Confocal immunofluorescence microscopy. A total $5 \times 10^{4}$ of HT29 and DLD1 cells were plated on glass coverslips or embedded in $0.5 \mathrm{mg} / \mathrm{ml}$ lr-ECM under $2 \mathrm{D}$ and $3 \mathrm{D}$ culture conditions, respectively, in 24-well plates. After $48 \mathrm{~h}$, cells were washed twice with phosphate-buffered saline (PBS) buffer and fixed with $4 \%$ paraformaldehyde (ROTH) solution in PBS at room temperature followed by three wash steps in PBS for $5 \mathrm{~min}$. Cell permeabilization was performed with ice-cold $0,1 \%$ Triton $\mathrm{X}-100$ in PBS for $3 \mathrm{~min}$. Cells were stained with Alexa ${ }^{\circledR} 633$ Phaloidin (ThermoFisher Scientific) in PBS containing 1\% bovine serum albumin for $30 \mathrm{~min}$. at room temperature. Next, coverslips were washed three times in PBS and counterstained with $5 \mu \mathrm{g} / \mathrm{ml}$ 4' 6 diamino-2-phenylindole (DAPI) (Sigma Aldrich, St. Louis, MO, USA) in PBS for $3 \mathrm{~min}$. Slides were rinsed three times in PBS and mounted with Roti ${ }^{\circledR}$-MountFluorCare mounting media (ROTH). Images were obtained using Zeiss LSM 7 Duo Live confocal microscope (Zeiss, Oberkochen, Germany) and $\times 40 / 1.3$ immersion objective and excitation wavelengths of $405 \mathrm{~nm}$ and $633 \mathrm{~nm}$. Confocal images shown are single optical slides.

Total RNA extraction. RNA was isolated from harvested cells using GeneJET RNA Purification Kit (ThermoFisher Scientific) according to manufacturer's instructions. The quantity and quality of RNA were evaluated using Nanodrop 2000c (ThermoFisher Scientific) and Bioanalyzer (Agilent Technologies, Santa Clara, CA, USA).

Global gene-expression analysis. cRNA sample preparation, labeling and hybridization were performed according to the manufacturer's instructions. Briefly, $1 \mu \mathrm{g}$ of total RNA was used for
cDNA synthesis and amplification using Message ${ }^{\text {TM }}$ Amp aRNA kit (ThermoFisher Scientific). Then $825 \mathrm{ng}$ of cRNA labeled with Cy3/Cy5 dyes using Arcturus ${ }^{\circledR}$ TURBO labeling ${ }^{\mathrm{TM}} \mathrm{Cy}^{\mathrm{TM}} 3 / \mathrm{Cy}^{\mathrm{TM}} 5$ Kit (Apllied Biosystems, Netherlands) were hybridized to Human 4x44k Oligonucleotide Microarrays (Agilent Technologies, USA) using HS 400 hybridization station (Tecan, Switzerland). Microarray slides were scanned using LS Reloaded scanner (Tecan, Switzerland) for microarray image analysis and data generated were further analyzed using ImaGene ver. 9.0 (BioDiscovery, USA) and GeneSpring GX ver. 11.0 software (Agilent Technologies, USA). Loess normalization was performed to adjust microarray data for variation. Gene expression fold change above 1.5 (with $p$-value $<0.05)$ was defined as differentially expressed between two conditions. KEGG pathway enrichment analysis was performed using Webgenstalt online source (15). All of the microarray data was deposited to GEO Dataset database, Accession number GSE75551 (http://www.ncbi.nlm.nih.gov/geo/query/acc.cgi?acc=GSE75551).

Real-time RT-PCR. A total of $1 \mu \mathrm{g}$ of RNA was used for cDNA synthesis using RevertAid RT Kit (ThermoFisher Scientific) according to manufacturer's instructions. Quantitative real-time polymerase chain reaction (PCR) was performed according to manufacturer's instructions. Briefly, for each reaction in a 96-well plate, $1 \mu \mathrm{l}$ cDNA, $2 \mu \mathrm{l}$ forward and reverse primer $(2 \mu \mathrm{M}), 10 \mu \mathrm{l}$ Maxima SYBR Green qPCR MasterMix (2X) (ThermoFisher Scientific) and $5 \mu \mathrm{l}$ nuclease-free water was used. The relative change of gene expression was calculated by the $\Delta \Delta \mathrm{Ct}$ method with hypoxanthine phosphoribosyltransferase 1 (HPRT1) as the gene used for sample normalization. Primers were synthesized by ThermoFisher Scientific (Table I).

\section{Results}

Cell morphology. Colon carcinoma DLD1 and HT29 cells were embedded in an Ir-ECM microenvironment and were inspected for altered cell morphology (Figure 1A). Both DLD1 and HT29 cells gained characteristic 'mass' morphology following $48 \mathrm{~h}$ of growth under lr-ECM 3D culture. Furthermore, in order to inspect morphological changes at increased resolution, we also performed confocal microscopy of cells by staining actin cytoskeleton with phaloidin (Figure 1B). The images indicated that CRC cells grown in 3D cell culture had undergone cytoskeleton rearrangement and lost actin stress fibers. 

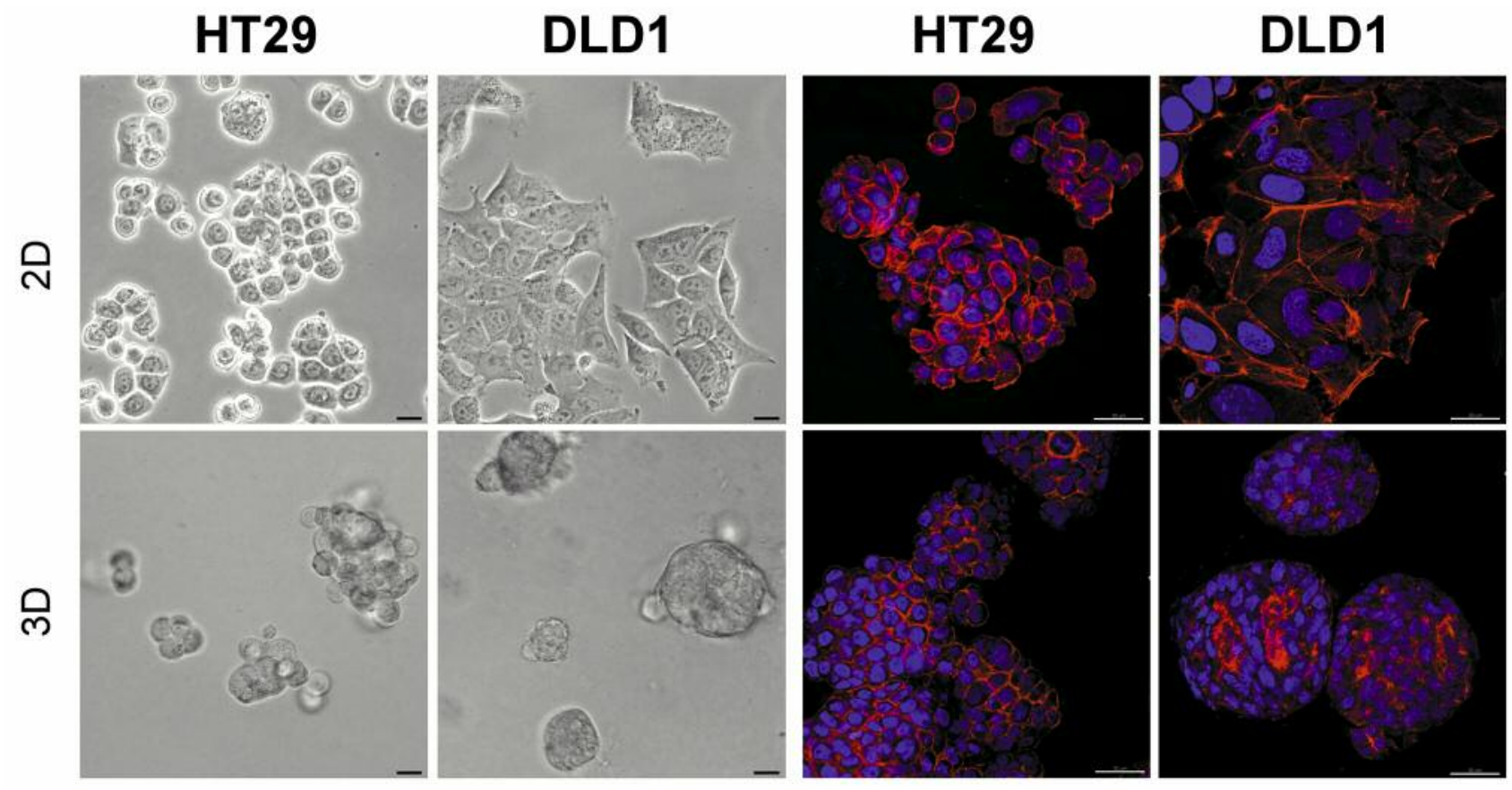

Figure 1. Cell morphology of DLD1 and HT29 cells grown under $2 D$ or laminin-rich extracellular matrix (lr-ECM) 3D cell culture conditions. Prior to imaging, cells were cultivated in $2 D$ (upper panel) and $3 D$ (lower panel) conditions for $48 \mathrm{~h}$. A: Representative phase-contrast images of cell growth in $2 D$ and $3 D$ cell culture. Bars $=50 \mu m$. B: Representative confocal laser scanning microscopy images of cell growth in $2 D$ and $3 D$ cell culture. F-Actin was stained with AlexaFluor 633 Phaloidin (red). Nuclei were counterstained with 4'6-diamino-2-phenylindole (DAPI) (blue). Bars $=50 \mu \mathrm{m}$.

Gene expression pattern. In order to evaluate the impact of the cellular microenvironment on gene-expression levels in DLD1 and HT29 CRC cells, we compared genome-wide expression levels in these cells following $48 \mathrm{~h}$ of growth in 2D and 1r-ECM 3D cell culture conditions. Microarray data revealed that the expression of 841 and 1190 genes was significantly altered ( $>1.5$ fold-change, $p<0.05)$ in an ECMdependent manner in DLD1 and HT29 cells, respectively (Figure 2). Most of these genes (637 in DLD1 cells and 804 in HT29 cells) were down-regulated. The Venn diagrams also revealed that the expression of 383 genes was significantly altered in both DLD1 and HT29 cells grown under 3D versus 2D conditions. However, in both cell lines, only 37 common genes were found to be up-regulated in contrast to 346 commonly down-regulated genes.

Pathway enrichment analysis. In order to better understand which biological processes were affected in an ECMdependent manner, we performed KEGG pathway enrichment analysis of differentially expressed genes in DLD1 and HT29 cells grown under 3D versus 2D culture conditions. Enrichment results revealed 45 and 35 significantly altered functional categories in DLD1 and HT29 cells, respectively. Table II presents 13 common functional categories significantly altered in both CRC cell lines. The "Metabolic pathways" category involved the highest number of differentially expressed genes (44 and 48 genes in DLD1 and HT29 cells) between 2D and 1r-ECM 3D. Nevertheless, KEGG pathway enrichment analysis revealed that the most significantly altered functional categories were related to cell-cell and cell-ECM interactions. The focal adhesion category was most significantly altered in CRC cells (DLD1: $p<7.47 \times 10^{-6}$; HT29: $p<1.59 \times 10^{-5}$ ) and resulted in significantly altered expression of 17 genes in DLD1 cells and 19 in HT29 cells. The present findings also indicated that functional categories adhesion junction, tight junction, ECM-receptor interaction and regulation of actin cytoskeleton were also prominently altered in an ECM-dependent manner. The majority of genes in these categories were down-regulated in CRC cells grown in 1 r-ECM 3D cell culture compared to 2D conditions. In addition, mitogen-activated protein kinase (MAPK) signaling pathway (DLD1: $p<0.0056$; HT29: $p<9.9 \times 10^{-7}$ ) was among the most significantly altered KEGG categories and comprised of 13 differentially regulated genes in DLD1 cells and 25 in HT29 cells. Inflammatory response-related categories significantly altered in both CRC cell lines were "chemokine signaling pathway" and "cytokine-cytokine 
Table II. Kyoto Encyclopedia of Genes and Genomes (KEGG) pathway enrichment analysis of genes differentially expressed in HT29 and DLDI cells between laminin-rich extracellular matrix (lr-ECM) 3D and $2 D$ cell culture conditions.

\begin{tabular}{|c|c|c|c|c|}
\hline \multirow[t]{2}{*}{ Functional category } & \multicolumn{2}{|c|}{ DLD-1 } & \multicolumn{2}{|c|}{ HT-29 } \\
\hline & Genes, $\mathrm{n}$ & $p$-Value & Genes, $\mathrm{n}$ & $p$-Value \\
\hline Focal adhesion & 17 & $7.47 \mathrm{e}-06$ & 19 & $1.59 \mathrm{e}-05$ \\
\hline MAPK signaling pathway & 13 & 0.0056 & 25 & $9.90 \mathrm{e}-07$ \\
\hline Pathways in cancer & 17 & 0.0005 & 25 & $1.59 \mathrm{e}-05$ \\
\hline Metabolic pathways & 44 & $1.95 \mathrm{e}-05$ & 48 & 0.0012 \\
\hline Adherens junction & 9 & 0.0001 & 10 & 0.0002 \\
\hline p53 signaling pathway & 8 & 0.0003 & 7 & 0.0092 \\
\hline Tight junction & 11 & 0.0003 & 10 & 0.0092 \\
\hline ECM-receptor interaction & 9 & 0.0003 & 7 & 0.0207 \\
\hline Regulation of actin cytoskeleton & 14 & 0.0003 & 18 & 0.0001 \\
\hline Endocytosis & 13 & 0.0005 & 17 & 0.0002 \\
\hline Chemokine signaling pathway & 11 & 0.0034 & 11 & 0.0247 \\
\hline Cytokine-cytokine receptor interaction & 12 & 0.0115 & 14 & 0.0207 \\
\hline WNT signaling pathway & 7 & 0.0387 & 9 & 0.0394 \\
\hline
\end{tabular}

MAPK: Mitogen-activated protein kinase. WNT: Wingless-type MMTV integration site family. Functional groups were enriched in at least five genes, $p<0.05$.

receptor interaction". Our results also indicated that p53 and wingless-type MMTV integration site family (WNT) signaling pathways were commonly altered in both cell lines. KEGG pathway classification also revealed that the number of differentially expressed genes in all categories and the significance and the extent of enrichment were similar in both cell lines. These findings indicate biologically related gene-expression changes during cellular adaptation to $1 \mathrm{r}$ ECM 3D cell culture conditions which could implicate cellular response to treatment and survival. All KEGG analysis data of differentially expressed genes in DLD1 and HT29 cells between 1r-ECM 3D and 2D conditions and complete list of genes associated with each category are given in supplementary tables (http://www.nvi.lt/wpcontent/uploads/2016/08/Supplementary_material.pdf).

Heat map analysis. ECM-induced expression changes of individual genes associated with the most significantly altered KEGG pathway categories, including cell adhesion, MAPK and inflammatory response subsets, were depicted to demonstrate expression patterns within each category subset (Figure 3). Figure 3A shows a heat map of a total of 59 genes from the cell adhesion subset, which includes genes involved in focal adhesion, adhesion junction, tight junction, ECM-receptor interaction and regulation of actin cytoskeleton functional categories. Although the expression of some individual genes did not pass the expression threshold (fold change $>1.5$ and $p<0.05$ ), the majority of genes from the cell adhesion subset were down-regulated in DLD1 and HT29 cells grown in 1r-ECM 3D compared with 2D cell culture conditions. Some of the down-regulated

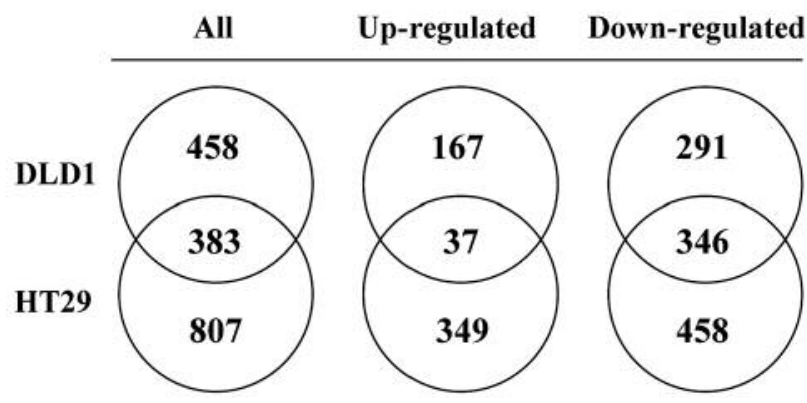

Figure 2. Venn diagrams showing the number of deregulated, upregulated and down-regulated differentially expressed genes (fold change of at least 1.5 and $p<0.05$ ) in HT29 and DLD1 cells following 48 hours growth under laminin-rich extracellular matrix (lr-ECM) $3 D$ and $2 \mathrm{D}$ cell culture conditions.

genes associated with cell-cell and cell-matrix junctions included integrins ITGA3, ITGA5, ITGB4, ITGB5 and ITGB7; filamins FLNA and FLNB; laminins LAMA3, LAMB2 and $L A M B 3$; and other structural genes including vinculin, actin $B$ and actinin 1, indicating changes in expression of genes closely related to the linkage between focal adhesion and the cytoskeleton during cellular adaptation to 1r-ECM 3D cell culture conditions. Additionally, the heat map data also revealed up-regulation of integrin ITGB8 in HT29 cells in an ECM-dependent manner.

Figure 3B depicts differentially expressed genes involved in the MAPK signaling pathway. In the MAPK category, the expression of 32 genes was altered in CRC cells grown 


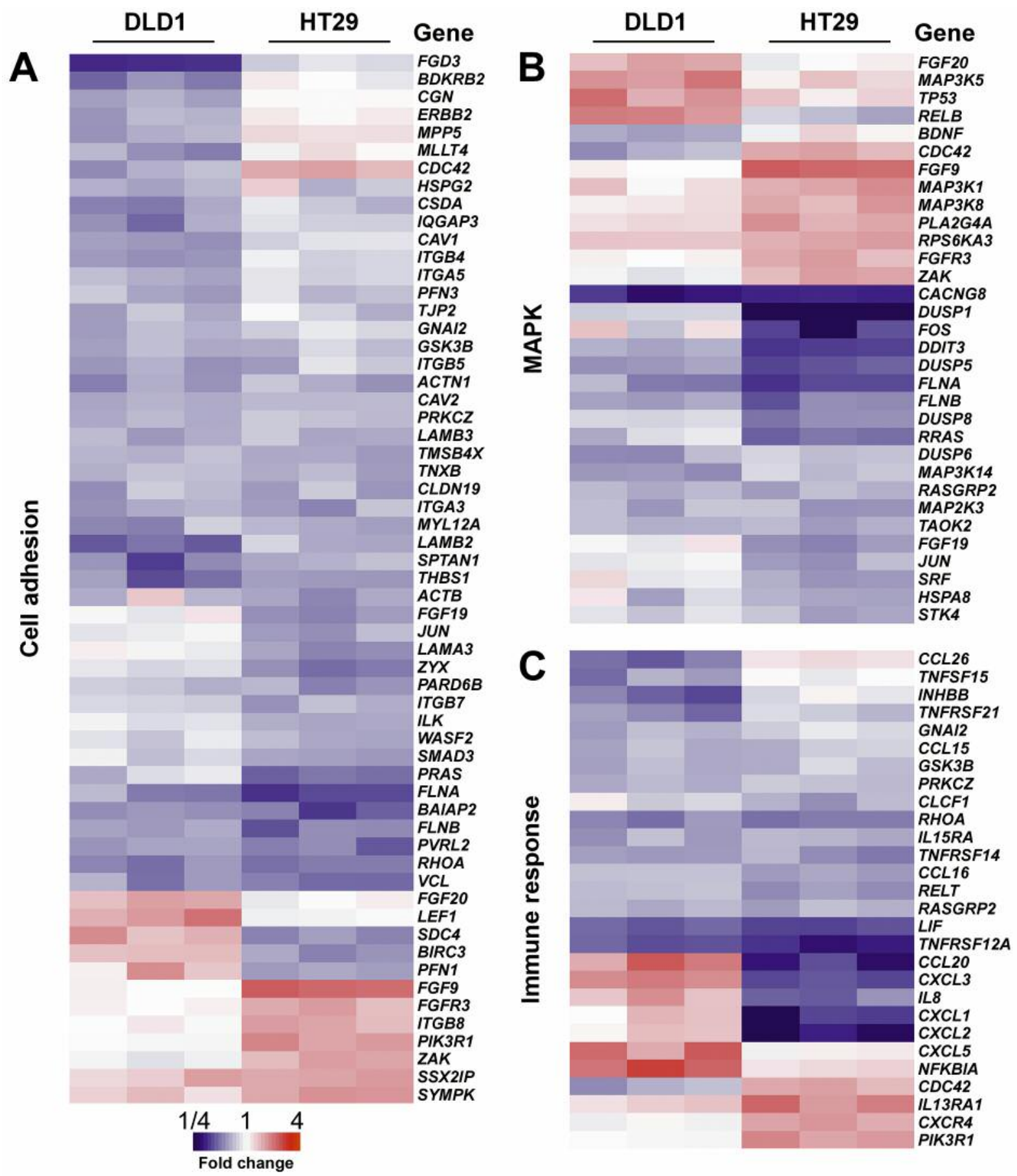

Figure 3. Heat map analysis of selected Kyoto Encyclopedia of Genes and Genomes (KEGG) categories. Heat maps representing the expression profile for cell adhesion (A), mitogen-activated protein kinase (MAPK) signaling (B), immune response (C) genes following $48 \mathrm{~h}$ of DLD1 and $H T 29$ cell growth under $3 D$ cell culture conditions compared to $2 D$. Red and blue indicate an increase and decrease of gene expression, respectively.

under lr-ECM 3D cell culture conditions compared to those under 2D. Differentially expressed genes included fibroblast growth factors $F G F 9, F G F 19$ and $F G F 20$, fibroblast growth factor receptor FGFR3; MAP kinase kinases MAP3K1,
$M A P 3 K 5, M A P 3 K 8, M A P 3 K 14$ and $M A P 2 K 3$, dual specificity phosphatases DUSP1, DUSP5, DUSP6 and DUSP8; and other kinases TAO kinase 2 (TAOK2) and serine/threonine kinase 4 (STK4). In addition, transcription 

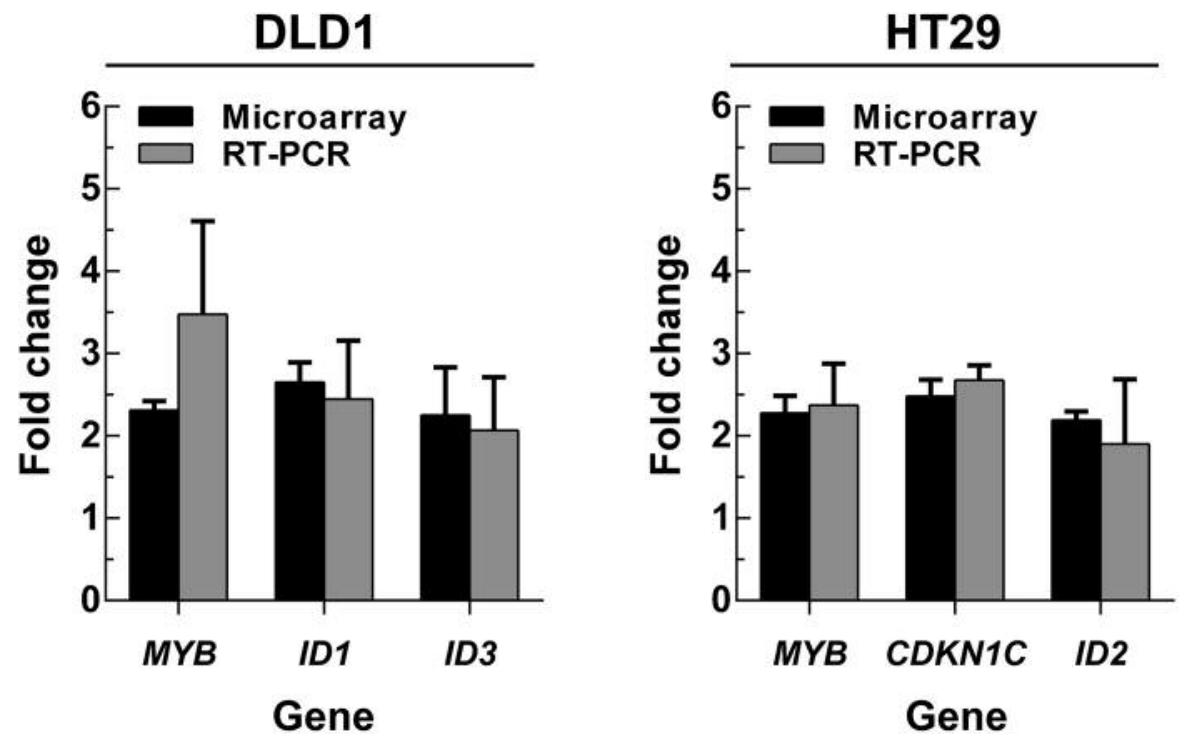

Figure 4. Validation of microarray gene expression data by quantitative polymerase chain reaction. qPCR was performed as described in the Materials and Methods section. The fold change in levels of selected genes (avian myeloblastosis oncogene (MYB), inhibitor of DNA binding 1 (ID1) and ID2 in DLD1; and MYB, cyclin-dependent kinase inhibitor $1 C$ (CDKN1C) and ID3 genes in HT29 cells, respectively) from microarray data after 48 hours of cultivation under laminin-rich extracellular matrix (lr-ECM) $3 D$ and $2 D$ cell culture conditions are shown. qPCR data analysis was based on $2^{-\triangle \Delta C t}$ and phosphoribosyltransferase 1 (HPRT1) was used as housekeeping gene for qPCR data normalization. Results show the mean $\pm S D(n=3)$.

factor AP1 subunits jun proto-oncogene $(J U N)$, murine osteosarcoma viral oncogene homolog $(F O S)$ and serum response factor $(S R F)$ were significantly down-regulated in HT29 cells in an ECM-dependent manner.

Figure 3C shows a heat map from the immune response subset which includes 28 differentially expressed genes involved in chemokine and cytokine-cytokine receptor signaling pathways. A total of 17 inflammatory genes were significantly down-regulated in one or both CRC cells and included chemokines CCL15, CCL16 and CCL26; tumor necrosis factor-related TNFSF 15, TNFRSF $12 a$, TNFRSF 14 and TNFRSF21; interleukin receptor IL15RA. C-X-C motif chemokine 5 (CXCL5), and chemokine receptor CXCR4 and interleukin receptor $I L 13 R A 1$ were significantly up-regulated in DLD1 and HT29 cells grown in 1r-ECM 3D. In addition, the heat map revealed an opposite expression pattern of five differentially expressed genes including chemokines CCL2O, $C X C L 1, C X C L 2, C X C L 3$ and interleukin IL8 was opposite between CRC cells. These genes were up-regulated in DLD1 cells and down-regulated in HT29 cells when CRC cells were grown under lr-ECM 3D cell culture conditions.

Microarray gene-expression data validation. In the present study, we observed that the genes most significantly upregulated in an ECM-dependent manner were involved in cellular differentiation maintenance. Thus, for microarray data validation, we performed expression analysis of inhibitor of
DNA binding $I D 1, I D 2$ and ID3; cyclin-dependent kinase inhibitor $C D K N 1 C$; and transcriptional activator $M Y B$ genes in CRC cells grown under 2D and $1 r-E C M$ 3D cell culture by real-time RT-PCR. The RT-PCR data confirmed the microarray data (Figure 4). The expression of most selected genes was up-regulated more than two-fold by RT-PCR.

\section{Discussion}

The loss of numerous physiological features was noted in cancer cells cultivated in 2D cell culture, leading to a poor representation of molecular events occurring in tumor tissue $(16,17)$. Nevertheless, restoration of cell characteristics physiologically more representative of tumor tissue, including cellular phenotype, gene expression and signaling patterns, is expected when cells are cultured in 3D (18). Observations that cancer cells grown under $3 \mathrm{D}$ cell culture conditions are less susceptible to agents of anticancer treatment suggest that differential gene expression profiles in cancer cells grown in 2D compared with $3 \mathrm{D}$ cell culture provide a therapeutic window for molecular targeting and prognostic strategies that could be used in the development of anticancer therapy. Hence, in the present study we compared cellular morphology and genome-wide expression changes in two CRC cell lines DLD1 and HT29 grown in lrECM 3D cell culture to cells grown on plastic. Furthermore, we also indicated the most significantly altered functional 
pathway categories in CRC cells cultured under 3D cell culture conditions.

In the present study, we observed that CRC cells grown under 1r-ECM 3D cell culture conditions compared to 2D displayed specific morphological changes as DLD1 and HT29 cells formed 3D spheroids. These observations are consistent with a previous report that demonstrated similarly altered cellular morphology of CRC cells cultured in 'ECM on top' 3D model (19). In addition, Kenny et al. also indicated that distinct 3D cell culture phenotypes of breast cancer cells reflected distinct gene-expression profiles correlating with invasiveness of tumor cell lines obtained from metastases (20). These observations suggest that ECM provides a more reliable microenvironment than plastic and is markedly more similar to the microenvironment in tumor tissue. Hence, further investigation of differential gene expression profiles using 3D cell culture models could uncover molecular characteristics of CRC cells closely related to tumors in vivo, which cannot be well established in $2 \mathrm{D}$ cell cultures.

Our microarray data demonstrated significantly altered expression signatures in CRC cells between the two cell culture models. We found that changes in cell culture conditions resulted in significantly altered expression of a total 841 and 1,190 genes in DLD1 and HT29 cells, respectively. Furthermore, our results revealed that a higher number of genes were down-regulated in an ECM-dependent manner compared to cells under 2D culture. In addition, microarray data also indicated 383 common genes differentially expressed in both cell lines, and most of these genes were also down-regulated in an ECM-dependent manner. These findings are consistent with previous reports that noted significantly altered gene-expression profiles in numerous cancer cell types between 2D and 3D cell culture models (19, 21-23). Furthermore, KEGG pathway enrichment results revealed ECM-dependent significantly altered gene functional categories in CRC cells grown in 2D and 3D cell cultures. We found that metabolic pathway, cell adhesion, p53 and WNT signaling, MAPK and immune response-related categories were significantly altered in both DLD1 and HT29 cells, suggesting the existence of common molecular mechanisms controlled in an ECM-dependent manner.

Our results indicate that functional categories associated with cell-ECM and cell-cell interactions were most significantly altered in CRC cells. In addition, microarray data revealed significantly altered expression of numerous integrins (ITGA3, ITGB4/5/7/8) in CRC cells grown under 3D and 2D cell culture conditions. These findings are in accordance with a previous report that also showed a differential expression of genes involved in the regulation of integrin signaling and cell-cell interaction, leading to different cellular response to radio- and chemotherapy (21).
In addition, Bissel et al.'s group also demonstrated that the inhibition of the integrin signaling cascade remarkably influenced the cellular phenotype and behavior of breast cancer cells cultured in 3D, The indicating the key role of the ECM-integrin signaling cascade during cellular adaptation to a 3D cell culture microenvironment (24).

Two previous studies demonstrated differential expression of MAPK pathway genes in CRC cells cultured under ECM 3D cell culture compared with $2 \mathrm{D}$ using genome-wide transcriptome approach. Tsunoda $e t$ al. revealed that activated $\mathrm{V}$-Ki-ras2 Kristen rat sarcoma viral oncogene homolog $(K R A S)$ in colon carcinoma HCT116 cells markedly suppressed DNA repair genes and apoptosis in a 3D cell culture but not in $2 \mathrm{D}$, suggesting a critical role of MAPK pathway in accumulation of genetic mutations through inhibition of tumor-suppressor genes (25). Luca et al. also demonstrated altered epidermis growth factor receptor (EGFR) protein levels and a switch between rat sarcoma oncogene RAS-MAPK pathway activation between 2D and 1r-ECM 3D models, suggesting that cellular adaptation to a 3D microenvironment might promote essential reorganization of molecular mechanisms to acquire resistance to targeted therapy during cancer progression (19). In keeping with previous considerations, our microarray results also indicated altered expression of fibroblast growth factor-related $F G F R 3$, $F G F 9 / 19 / 20$ and MAPK kinases MAP2K3, MAP3K1, $M A P 3 K 5, M A P 3 K 8$ and MAP $3 K 14$ genes in CRC cells grown under $3 \mathrm{D}$ and $2 \mathrm{D}$ cell culture conditions.Together these observations suggest that the ECM plays crucial role in regulation of MAPK pathway-related genes. Thus, 3D culture model as a useful approach could promote the investigation of MAPK-driven molecular mechanisms in CRC development.

Additionally, microarray data also revealed differential expression of genes associated with inflammatory response in CRC cells grown under 1r-ECM 3D and 2D conditions. We found that the expression of genes related to cytokine (e.g. IL8) and chemokine (e.g. CXCL1-3) signaling pathways were markedly altered in an ECM-dependent manner. Chemokines and cytokines play key roles in the initiation of immune response during tumor development (26). Chemokines attract lymphocytic migration, whereas cytokines can direct the polarization and activation of antigen-presenting cells (e.g. macrophages) and T-cells. Nevertheless, deregulated signaling of cytokines and chemokines in a tumor microenvironment promotes tumor progression (27). In keeping with these observations, we suggest that the 3D cell culture model should be considered an essential tool for investigating genes involved in tumor cell-immune system interactions. Better understanding of the cross-talk between tumor and immune cells in an ECMdependent manner could also notably promote development of preclinical targeted immunotherapy which cannot be properly established under 2D culture conditions. 
Finally, we also validated up-regulation of $M Y B$ and $I D I$, ID2 and ID3 genes in CRC cells grown under 3D compared with those under $2 \mathrm{D}$ cell culture conditions by qPCR. The transcription factor $M Y B$ and ID family genes play critical roles in determine a fate of stem and progenitor cell fate during normal development and lifespan. Nevertheless, deregulated expression of these genes is detected in many types of tumors $(28,29)$. Overexpression of $M Y B$ in tumor cells promotes cellular expansion and self-renewal. In addition, ID proteins are implicated not only in maintaining cancer stem cells but also in many other cancer developmentrelated molecular mechanisms (e.g. tumor angiogenesis or metastasis) (29). Furthermore, high expression of $M Y B$ and ID genes in tumors correlates with poor survival prognosis, suggesting these genes as plausible prognostic and therapeutic targets $(30,31)$. Consequently, in accordance with these observations, our findings suggest that CRC cells recapitulate clinically relevant oncogenic properties in $3 \mathrm{D}$ cell culture conditions more similar to those of tumor tissue and reveal key features for the development of targeted therapies.

In summary, we revealed differential gene expression signatures in two human CRC DLD1 and HT29 cell lines grown in 2D and 3D cell culture conditions. Our results defined characteristic cancer pathways related to cell-ECM interaction, immune response, proliferation and differentiation, which were significantly altered in both cell lines in an ECM-dependent manner. KEGG pathway enrichment analysis indicated that cell adhesion, MAPK and immune response-related categories were significantly altered in both DLD1 and HT29 cells, suggesting the existence of common molecular mechanisms controlled in an ECM-dependent manner. Our findings suggest that pathways important for cancer development and progression are altered during the shift from 2D to 3D cell culture and reveal potential targets for the development of anticancer therapies using 3D ECM-enriched models.

\section{Competing Interests}

None of the Authors have any competing interests.

\section{Acknowledgements}

This research was funded by a grant No. MIP-028/2014 from the Research Council of Lithuania.

\section{References}

1 Griffith LG and Swartz MA: Capturing complex 3D tissue physiology in vitro. Nat Rev Mol Cell Biol 7(3): 211-224, 2006.

2 Yamada KM and Cukierman E: Modeling tissue morphogenesis and cancer in 3D. Cell 130(4): 601-610, 2007.

3 Bonnans C, Chou J and Werb Z: Remodelling the extracellular matrix in development and disease. Nat Rev Mol Cell Biol 15(12): 786-801, 2014.
4 Frith JE, Thomson B and Genever PG: Dynamic threedimensional culture methods enhance mesenchymal stem cell properties and increase therapeutic potential. Tissue Eng Part C Methods 16(4): 735-749, 2010.

5 Wolf $\mathrm{K}$ and Friedl P: Extracellular matrix determinants of proteolytic and non-proteolytic cell migration. Trends Cell Biol 21(12): 736-744, 2011.

6 Rayavarapu RR, Heiden B, Pagani N, Shaw MM, Shuff S, Zhang SY and Schafer ZT: The role of multicellular aggregation in the survival of ERBB2-positive breast cancer cells during extracellular matrix detachment. J Biol Chem 290(14): 87228733, 2015.

7 Schrader J, Gordon-Walker TT, Aucott RL, van Deemter M, Quaas A, Walsh S, Benten D, Forbes SJ, Wells RG and Iredale JP: Matrix stiffness modulates proliferation, chemotherapeutic response, and dormancy in hepatocellular carcinoma cells. Hepatology 53(4): 1192-1205, 2011.

8 Nguyen-Ngoc KV, Cheung KJ, Brenot A, Shamir ER, Gray RS, Hines WC, Yaswen $P$, Werb $Z$ and Ewald AJ: Ecm microenvironment regulates collective migration and local dissemination in normal and malignant mammary epithelium. Proc Natl Acad Sci USA 109(39): E2595-E2604, 2012.

9 Helleman J, Jansen MPHM, Burger C, van der Burg MEL and Berns EMJJ: Integrated genomics of chemotherapy resistant ovarian cancer: A role for extracellular matrix, TGFbeta and regulating micrornas. Int $\mathrm{J}$ Biochem Cell Biol 42(1): 25-30, 2010.

10 Eke I and Cordes N: Radiobiology goes 3d: How ecm and cell morphology impact on cell survival after irradiation. Radiother Oncol 99(3): 271-278, 2011.

11 Eke I, Dickreuter E and Cordes N: Enhanced radiosensitivity of head and neck squamous cell carcinoma cells by $\beta 1$ integrin inhibition. Radiother Oncol 104(2): 235-242, 2012.

12 Li QW, Chow AB and Mattingly RR: Three-dimensional overlay culture models of human breast cancer reveal a critical sensitivity to mitogen-activated protein kinase kinase inhibitors. J Pharm Exp Ther 332(3): 821-828, 2010.

13 Birgersdotter A, Sandberg R and Ernberg I: Gene expression perturbation in vitro - a growing case for three-dimensional (3D) culture systems. Semin Cancer Biol 15(5): 405-412, 2005.

14 Eke I, Hehlgans S, Sandfort V and Cordes N: 3D matrix-based cell cultures: Automated analysis of tumor cell survival and proliferation. Int J Oncol 48(1): 313-321, 2016.

15 Wang J, Duncan D, Shi Z and Zhang B: Web-based gene set analysis toolkit (webgestalt): Update 2013. Nucleic Acids Res 41(W1): W77-W83, 2013.

16 Irish JM, Hovland R, Krutzik PO, Perez OD, Bruserud O, Gjertsen BT and Nolan GP: Single-cell profiling of potentiated phospho-protein networks in cancer cells. Cell 118(2): 217-228, 2004.

17 Ross DT, Scherf U, Eisen MB, Perou CM, Rees C, Spellman P, Iyer V, Jeffrey SS, Van de Rijn M, Waltham M, Pergamenschikov A, Lee JCE, Lashkari D, Shalon D, Myers TG, Weinstein JN, Botstein D and Brown PO: Systematic variation in gene expression patterns in human cancer cell lines. Nat Gen 24(3): 227-235, 2000.

18 Storch K, Eke I, Borgmann K, Krause M, Richter C, Becker K, Schrock E and Cordes N: Three-dimensional cell growth confers radioresistance by chromatin density modification. Cancer Res 70(10): 3925-3934, 2010 
19 Luca AC, Mersch S, Deenen R, Schmidt S, Messner I, Schafer KL, Baldus SE, Huckenbeck W, Piekorz RP, Knoefel WT, Krieg $\mathrm{A}$ and Stoecklein NH: Impact of the 3D microenvironment on phenotype, gene expression, and egfr inhibition of colorectal cancer cell lines. PLoS One 8(3): e59689, 2013.

20 Kenny PA, Lee GY, Myers CA, Neve RM, Semeiks JR, Spellman PT, Lorenz K, Lee EH, Barcellos-Hoff MH, Petersen OW, Gray JW and Bissell MJ: The morphologies of breast cancer cell lines in three-dimensional assays correlate with their profiles of gene expression. Mol Oncol 1(1): 84-96, 2007.

21 Zschenker O, Streichert T, Hehlgans S and Cordes N: Genomewide gene expression analysis in cancer cells reveals $3 \mathrm{D}$ growth to affect ECM and processes associated with cell adhesion but not DNA repair. PLoS One 7(4): e34279, 2012.

22 Fournier MV and Martin KJ: Transcriptome profiling in clinical breast cancer: From 3D culture models to prognostic signatures. J Cell Physiol 209(3): 625-630, 2006.

23 Härmä V, Virtanen J, Mäkelä R, Happonen A, Mpindi J-P, Knuuttila M, Kohonen P, Lötjönen J, Kallioniemi O and Nees M: A comprehensive panel of three-dimensional models for studies of prostate cancer growth, invasion and drug responses. PLoS One 5(5): e10431, 2010.

24 Weaver VM, Petersen OW, Wang F, Larabell CA, Briand P, Damsky $\mathrm{C}$ and Bissell MJ: Reversion of the malignant phenotype of human breast cells in three-dimensional culture and in vivo by integrin blocking antibodies. J Cell Biol 137(1): 231-245, 1997.

25 Tsunoda T, Takashima Y, Fujimoto T, Koyanagi M, Yoshida Y, Doi K, Tanaka Y, Kuroki M, Sasazuki T and Shirasawa S: Threedimensionally specific inhibition of DNA repair-related genes by activated KRAS in colon crypt model. Neoplasia (New York, NY) 12(5): 397-404, 2010.
26 Burkholder B, Huang R-Y, Burgess R, Luo S, Jones VS, Zhang W, Lv Z-Q, Gao C-Y, Wang B-L, Zhang Y-M and Huang R-P: Tumor-induced perturbations of cytokines and immune cell networks. BBA-Rev Cancer 1845(2): 182-201, 2014.

27 Zamarron BF and Chen W: Dual roles of immune cells and their factors in cancer development and progression. Int J Biol Sci 7(5): 651-658, 2011.

28 Ramsay RG and Gonda TJ: Myb function in normal and cancer cells. Nat Rev Cancer 8(7): 523-534, 2008.

29 Lasorella A, Benezra R and Iavarone A: The id proteins: Master regulators of cancer stem cells and tumour aggressiveness. Nat Rev Cancer 14(2): 77-91, 2014.

30 Gray MJ, Dallas NA, Van Buren G, Xia L, Yang AD, Somcio RJ, Gaur P, Mangala LS, Vivas-Mejia PE, Fan F, Sanguino AM, Gallick GE, Lopez-Berestein G, Sood AK and Ellis LM: Therapeutic targeting of ID2 reduces growth of human colorectal carcinoma in the murine liver. Oncogene 27(57): 7192-7200, 2008.

31 Cross RS, Malaterre J, Davenport AJ, Carpinteri S, Anderson RL, Darcy PK and Ramsay RG: Therapeutic DNA vaccination against colorectal cancer by targeting the MYB oncoprotein. Clin Transl Immunology 4(1): e30, 2015.

Received July 28, 2016

Revised August 12, 2016

Accepted August 18, 2016 